\title{
Finalités de l'enseignement de la poésie au secondaire québécois
}

Purpose of teaching poetry in high schools in Quebec

Judith Émery-Bruneau

\section{(2) OpenEdition}

\section{Journals}

Édition électronique

URL : http://journals.openedition.org/pratiques/4747

DOI : $10.4000 /$ pratiques. 4747

ISSN : 2425-2042

Éditeur

Centre de recherche sur les médiations (CREM)

Référence électronique

Judith Émery-Bruneau, «Finalités de l'enseignement de la poésie au secondaire québécois », Pratiques [En ligne], 179-180 | 2018, mis en ligne le 31 décembre 2018, consulté le 14 novembre 2019. URL http://journals.openedition.org/pratiques/4747 ; DOI : 10.4000/pratiques.4747

Ce document a été généré automatiquement le 14 novembre 2019.

(c) Tous droits réservés 


\title{
Finalités de l'enseignement de la poésie au secondaire québécois
}

\author{
Purpose of teaching poetry in high schools in Quebec
}

\author{
Judith Émery-Bruneau
}

1 Dans les prescriptions officielles du secondaire québécois, comme dans celles du primaire d'ailleurs, les textes de genre poétique doivent être enseignés pendant les cinq années, contrairement, par exemple, aux contes et légendes prescrites spécifiquement pour la $3^{\mathrm{e}}$ année du secondaire ou au théâtre et à la nouvelle en $4^{\mathrm{e}}$ année. Commune à toutes les années de scolarisation obligatoire, la poésie présente un angle d'analyse indéniable pour comprendre la progression des objets d'enseignement. Or, si quelques didacticiens se sont intéressés à la poésie comme genre facilitant le développement des sujets lecteurs (Brillant-Rannou, 2010, 2016; Favriaud, 2011; Boutevin, 2014), permettant de travailler la langue du dire-lire-écrire (Martin, 2010; Favriaud, Vinsonneau \& Poletto, 2017) ou articulant l'oral à l'écrit dans le cadre de performances poétiques (Brunel \& Émery-Bruneau, 2016; Émery-Bruneau \& Brunel, 2016), l'enseignement de la poésie sous l'angle de la progression des objets d'enseignement qui y sont liés a fait l'objet de peu de recherches jusqu'à présent. Pourtant, l'étude de la progression est une question clé de la didactique (Nonnon, 2010), influencée notamment par les transformations des conditions de scolarisation, le statut des savoirs et les pratiques des enseignants.

2 Nous avons alors voulu réfléchir à la notion de poésie en tant qu'objet d'enseignement pour mieux en comprendre les croisements, les ruptures et les continuités entre la vision des didacticiens de la littérature, la vision institutionnelle émanant des programmes et manuels, ainsi que les pratiques d'enseignement des passeurs de poésie. Car là où tous les discours se rejoignent, c'est sur le fait que les conceptions de la poésie se développent rarement en dehors de la forme scolaire. En effet, alors que nous avions interrogé des étudiants en formation initiale à l'enseignement au secondaire dans le cadre d'une autre recherche (Émery-Bruneau \& Leclerc, 2018), l'un d'eux affirmait: "J'ai longtemps pensé que la poésie devait nécessairement être organisée en vers symétriques contenant des rimes. Cette conception stéréotypée a pris naissance à 
l'école, dans les classes de français ». Or, le discours de ce futur enseignant conscient que c'est l'école qui a forgé sa manière de concevoir la poésie croise celui des enseignants en exercice que nous avions interrogés pour connaitre leur rapport à la poésie (Émery-Bruneau, 2018). Par exemple, l'un des 20 enseignants interrogés pour cette recherche ${ }^{1}$ avait souligné qu'il est important d'enseigner la poésie au secondaire " [parce que] c'est potentiellement le seul contact que les élèves auront avec la poésie ». Ces discours actuels de futurs enseignants et d'enseignants d'expérience croisent d'ailleurs celui des didacticiens qui affirmaient il y a 20 ans déjà que « c'est sans doute à l'école qu'on lit le plus de poésie et, une fois hors d'elle, on n'en lit guère » (Canvat \& Legros, 1997, p. 5). Il semble donc que tous les acteurs partagent un même constat: l'école serait le principal lieu où la poésie est consommée ou expérimentée, même si les enseignants se sentent plus ou moins confortables avec cet objet (Duval \& Turcotte, 2007), et même si les pratiques semblent cristallisées dans des approches traditionnelles d'imitation, de récitation et de lecture expliquée (Émery-Bruneau, 2018). Cela dit, quelques pratiques d'enseignement alternatives défient néanmoins cette cristallisation et présentent un pont entre les pratiques ordinaires et les innovations des didacticiens: nous les soulignons dans les dernières parties de cet article.

\section{La poésie : points de vue des didacticiens de la littérature}

3 Dans leur ouvrage qui expose les liens entre la didactique et les pratiques poétiques, M. Favriaud, M. Vinsonneau et M. Poletto $(2017$, p. 8) ancrent la poésie au cœur de l'actualité didactique, la définissant comme le «lieu (sensible, esthétique, peut-être philosophique) d'interrogation du rapport au monde et à la langue, et sans doute aussi le lieu critique (l'envers complémentaire) de la didactique du français ». Or, comme le rappelaient K. Canvat et G. Legros (1997, p. 23), « la première exigence, pour toute discipline scolaire, est d'identifier clairement son objet propre.» Alors comment les didacticiens de la littérature conçoivent-ils la poésie en tant qu'objet d'enseignement ?

M. Collot (2005), un poéticien phénoménologue qui a travaillé du côté des émotions qu'évoque la poésie à travers l'expérience poétique engageant le sujet, a nourri la réflexion des didacticiens. Par exemple, dans un chapitre consacré au genre poétique, J.-L. Dufays, M. Lisse et C. Meurée (2009, p. 77) avancent que la poésie est une « matière émotion", c'est-à-dire "une émotion faite langue, un condensé de lyrisme et de matérialisme, d'expérience humaine et de travail langagier ». Cette perspective n'est pas sans rappeler les dimensions plus générales du phénomène littéraire que J.L. Dufays, L. Gemenne et D. Ledur (2015) définissent ailleurs comme art du verbe, mise en scène de l'expérience humaine, construction historique et sociale, et travail du lecteur comme principal agent d'actualisation du texte littéraire. Or, N. BrillantRannou (2010, p.31), dans l'introduction de sa thèse, rappelle qu'il est «tentant de songer que "la poésie est partout et nulle part" (Delas, 1973, p. 8), [m]ais cette dilution de la poésie ne permet pas de rendre compte de nos pratiques de lecture concrètes et effectives » des textes de genres poétiques.

5 Mais alors, qu'est-ce qui s'avèrerait spécifique à la poésie dans le domaine de l'enseignement de la littérature? Par exemple, pour le meschonnicien et didacticien S. Martin (2010, p. 12), la poésie se trouve dans le langage, mais aussi chez le sujet du 
poème, soit le sujet créateur ou lecteur (réénonciateur) tel qu'il advient par le poème présent et tel que celui-ci transforme le sujet antérieur, car le sujet n'est pas seulement tourné vers son passé identitaire, mais aussi vers son futur marqué d'inattendus, de rencontres : «La finalité d'un enseignement de la poésie est donc à chercher dans une éthique du langage qui met le personnel dans l'échange, le plaisir dans l'écoute, le gain dans l'inconnu, le savoir dans la surprise, l'inattendu dans le travail et surtout la voix (voie) dans le poème.» (ibid., p. 12). En ce sens, M. Favriaud, M. Vinsonneau et M. Poletto (2017) associent aussi la poésie à l'usage de la langue, au corps-émotion et à la voix. Pour N. Brillant-Rannou (2010), la poésie renvoie aux expériences d'un sujet marqué d'une émotion poétique. Pour elle, la poésie est une « expérience de lecture, et cet événement peut avoir lieu avec tout type de poésie, quelles qu'en soient l'époque et l'esthétique, quelles que soient les distorsions interprétatives que lui fera vivre le lecteur " (ibid., p. 29), bref, c'est l'émotion poétique. En tenant compte des expériences poétiques des sujets lecteurs, mais aussi scripteurs et performeurs qu'on ne peut désormais plus ignorer en didactique, rappelons qu'il y a 20 ans, K. Canvat et $\mathrm{G}$. Legros (1997, p. 26) défendaient qu'un "véritable enseignement de la poésie devrait [aussi] viser à former des lecteurs connaisseurs et pas seulement des amateurs, et que de tels lecteurs ont besoin de connaissances même sur des œuvres qu'ils n'ont pas lues ». Dans cette perspective, les pratiques d'enseignement de la poésie devraient prendre en compte, en plus des expériences du sujet, des savoirs sociaux (le champ littéraire et ses conflits de valeurs, le paratexte, l'image des poètes, les modes de lecture, etc.), techniques (savoirs syntaxiques, sémantiques, métriques, génériques, rhétoriques, narratologiques - le cas échéant -, typographiques, etc.) et historiques (contexte de production, courant littéraire, etc.).

Nous retenons donc que, dans une perspective essentiellement didactique, la poésie en tant qu'objet d'enseignement inclus à la fois un langage (écrit-oral), des performances vocales et corporelles (entendues/visionnées, produites), des savoirs (sociaux, langagiers, historiques) et des genres poétiques variés (poème lyrique ou engagé, chanson, slam, etc.) déterminés par le champ de production (maisons d'édition de poésie, manifestations poétiques, rôle social, politique ou linguistique du poète, etc.) et de réception (expériences poétiques singulières de sujets lecteurs, scripteurs, auditeurs, spectateurs et/ou performeurs).

\section{Méthodologie : entre analyse de discours et analyse de contenu}

7 Pour faire l'analyse des discours institutionnels et celui des passeurs de poésie, nous avons utilisé trois types de données, lesquelles ont fait l'objet d'une analyse de contenu pour dégager les discours dominants.

8 Nous avons analysé les prescriptions officielles de l'enseignement du français du secondaire québécois (MÉQ, 2005 ; MÉLS, 2009, 2011). Notre focale n'était orientée que sur les aspects touchant spécifiquement à la poésie et aux genres poétiques. Nous avions pour but d'identifier toutes les composantes de l'objet " poésie », en regard des trois compétences, soit « lire et apprécier des textes variés ", « écrire des textes variés " et « communiquer oralement selon des modalités variées ». 
9 Nous avons également analysé tous les manuels ${ }^{2}$ de la fin du $1^{\text {er }}$ cycle $\left(2^{\mathrm{e}}\right.$ année du secondaire) et de la fin du $2^{\mathrm{e}}$ cycle ( $5^{\mathrm{e}}$ et dernière année du secondaire). Nous avons donc analysé ce corpus de huit manuels et l'avons soumis à trois questions. Au niveau macro d'abord, nous avons quantifié le nombre de pages consacrées à la poésie dans ces manuels destinés à l'enseignement du français et examiné les corpus de textes poétiques. Au niveau micro ensuite, nous avons cerné la didactisation de la poésie en répondant aux questions suivantes: 1- Quels sont les savoirs convoqués et dans quel but?; 2- Quelles tâches privilégie-t-on pour travailler la poésie et pour former quel type de lecteur, scripteur, auditeur et/ou performeur?

Enfin, pour connaitre les pratiques d'enseignement de la poésie, nous nous appuyons sur les résultats tirés d'entretiens semi-dirigés d'une durée approximative d'une heure chacune, réalisées avec 20 enseignants, dont quatre à cinq personnes par degré. Les résultats présentés portent sur cinq aspects : les savoirs enseignés dans leurs séquences sur la poésie, les tâches des élèves, les finalités poursuivies, les limites qui freinent leurs enseignements et les pratiques alternatives exercées par quelques-uns d'entre eux.

\section{Forme scolaire et enseignement de la poésie : croisement des discours}

11 Dans cette section, nous présentons nos analyses de contenu des trois discours (programmes, manuels et enseignants).

\section{Le discours officiel dans les programmes du secondaire québécois}

12 Trois documents officiels québécois sont prescriptifs quant aux genres et savoirs à enseigner selon les degrés (MÉQ, 2005 ; MÉLS, 2009, 2011). La poésie doit être travaillée dans les trois compétences langagières, bien que les élèves ne doivent s'inscrire que dans une posture d'écoute en ce qui concerne l'oral.

\section{Genres poétiques prescrits}

Les genres poétiques ont été sélectionnés en fonction des autres genres et modes de discours prescrits selon les degrés; la poésie est parfois utilisée comme un moyen pour travailler un mode de discours (descriptif, argumentatif, etc.) plutôt que comme objet. Par exemple, les poèmes à trame narrative ou descriptive sont prescrits pour le $1^{\mathrm{er}}$ cycle, car il s'agit des séquences textuelles que les enseignants doivent aussi travailler avec des textes non littéraires comme l'article de journal ou la biographie. Puis, au $2^{\mathrm{e}}$ cycle, alors que les élèves apprennent à argumenter, à participer à des débats délibératifs et à produire des textes d'opinion, la poésie engagée est le genre sélectionné en raison de certaines caractéristiques communes à ces autres genres, dont la modalisation et le point de vue énonciatif. Le tableau 1 présente les genres poétiques prescrits (MELS, 2011, p. 37-42). Il s'agit des seules indications quant aux textes à travailler, car aucun corpus ni auteur ne sont imposés dans les programmes québécois. 
Tableau 1. Genres poétiques à enseigner au secondaire québécois

\begin{tabular}{|c|c|c|c|c|}
\hline & degrés ${ }^{3}$ & LECTURE & ÉCRITURE & ORAL (ÉCOUTE) \\
\hline \multirow{2}{*}{$\begin{array}{l}1^{\text {er }} \\
\text { cycle }\end{array}$} & $1^{\mathrm{re}}$ & $\begin{array}{l}\text { poème ludique } \\
\text { et poème ayant une } \\
\text { trame descriptive ou } \\
\text { narrative }\end{array}$ & $\begin{array}{l}\text { jeux poétiques sur les mots, sur } \\
\text { les sons, sur les formes }\end{array}$ & \multirow{2}{*}{$\begin{array}{l}\text { poème ludique } \\
\text { ou poème ayant une } \\
\text { trame descriptive ou } \\
\text { narrative }\end{array}$} \\
\hline & $2^{\mathrm{e}}$ & $\begin{array}{l}\text { poème ayant une } \\
\text { trame descriptive ou } \\
\text { narrative }\end{array}$ & $\begin{array}{l}\text { poème original ou transformé à } \\
\text { partir de procédés imposés ou } \\
\text { choisis }\end{array}$ & \\
\hline \multirow{3}{*}{$\begin{array}{l}2^{\mathrm{e}} \\
\text { cycle }\end{array}$} & $3^{e}$ & poème lyrique & $\begin{array}{l}\text { poème original, transformé, } \\
\text { chanson qui évoque des } \\
\text { émotions, des sentiments, un état } \\
\text { d'âme... }\end{array}$ & $\begin{array}{l}\text { poème ou chanson } \\
\text { lyrique }\end{array}$ \\
\hline & $4^{\mathrm{e}}$ & $\begin{array}{l}\text { poème lyrique } \\
\text { et poème engagé }\end{array}$ & \multirow{2}{*}{ poème engagé } & $\begin{array}{l}\text { poème, chanson } \\
\text { engagée ou slam }\end{array}$ \\
\hline & $5^{e}$ & $\begin{array}{l}\text { poème classique ou } \\
\text { moderne, lien avec } \\
\text { culture et société }\end{array}$ & & $\begin{array}{l}\text { poème ou chanson, } \\
\text { lien avec culture et } \\
\text { société }\end{array}$ \\
\hline
\end{tabular}

La progression est à la fois pensée en fonction des genres, de leurs caractéristiques textuelles, grammaticales et sémantiques et de son appropriation par les élèves. Du côté de l'enseignant, on s'attend à ce qu'il soit un passeur culturel capable de proposer des expériences poétiques variées et d'accompagner les élèves dans leur découverte.

\section{Vision de la poésie dans les discours institutionnels}

Dans les programmes, on veut initier les élèves à la poésie, tant par les visées d'exploration et de découverte (dans les postures de réception) que d'expérimentation (en écriture). La formation des élèves comme sujets semble en outre une finalité, car il est prescrit de les amener à se construire des repères culturels en fonction des genres poétiques variés qu'ils sont appelés à lire ou à écouter/visionner. La progression est donc pensée de la familiarisation ludique chez les plus jeunes à l'engagement du sujet vers la fin de la scolarité obligatoire, car elle est d'abord présentée par le jeu avec le langage au primaire et au $1^{\text {er }}$ cycle du secondaire, puis dans une dimension psychoaffective (analyse ou expression des émotions en $3^{\mathrm{e}}$ secondaire, avec les poèmes et chansons lyriques), à un objet plus intellectualisé dans les deux dernières années (analyse ou productions de poésie engagée).

\section{Le discours dans les manuels}

L'analyse des manuels porte sur trois aspects: les corpus, les savoirs et les tâches privilégiés pour travailler la poésie. 


\section{Les corpus}

17 L'espace accordé à la poésie est mince dans tous les manuels : au $1^{\text {er }}$ cycle, $8 \%$ des pages en moyenne portent sur la poésie alors qu'au $2^{\mathrm{e}}$ cycle, c'est $10 \%$. Les corpus sont surtout québécois (plus nombreux au $1^{\mathrm{er}}$ cycle) et français (plus nombreux au $2^{\mathrm{e}}$ cycle), auxquels se greffent quelques textes de poètes japonais, haïtiens, sénégalais, algériens, amérindiens... Les époques les plus représentées sont les $\mathrm{XIX}^{\mathrm{e}}$ et $\mathrm{XX}^{\mathrm{e}}$ siècles et les genres apparaissant systématiquement sont la fable, le haiku, le calligramme et le sonnet. La chanson est en outre très présente, ce qui pourrait s'expliquer par le fait qu'elle soit plus accessible pour les élèves, faisant partie de leurs référents culturels. Quant aux auteurs, É. Nelligan, poète québécois du début du $\mathrm{XX}^{\mathrm{e}}$ siècle, se trouve dans tous les manuels, ainsi que G. Apollinaire et J. de La Fontaine qui figurent dans la plupart des manuels. La principale distinction entre les cycles est liée à la dichotomie entre chanteurs contemporains, très présents dans les manuels du $1^{\mathrm{er}}$ cycle, et auteurs canonisés au $2^{\mathrm{e}}$ cycle, dont les plus récurrents sont P. Verlaine, J. Prévert, V. Hugo, A. Rimbaud et C. Baudelaire, ainsi que les québécois A. Hébert, H. de Saint-DenysGarneau et R. Lévesque.

\section{Les savoirs}

Les savoirs présentés, peu importe les cycles, sont de deux types : sociohistorique, par la présentation de courtes notices biographiques sur les auteurs ou pour décrire le contexte de production du poème; langagier, par le travail sur le lexique et sur quelques notions de grammaire, ainsi que par l'analyse de la forme des poèmes (règles de versification, rimes) et des figures de style. La seule distinction concerne les caractéristiques liées à l'argumentation (thèse, argument, point de vue) qui n'apparaissent que dans les manuels du $2^{\mathrm{e}}$ cycle, puisqu'il s'agit aussi de notions liées au texte d'opinion et à la lettre ouverte, genres travaillés à la dernière année du secondaire et faisant l'objet d'un examen national certificatif.

\section{Les tâches}

19 En lecture, on demande parfois aux élèves de nommer leurs émotions, réactions et appréciations d'un poème, mais le plus souvent, il leur est plutôt demandé de construire le sens du texte à partir du lexique (définir un mot ou le sens d'une expression), des thèmes, d'une analyse de la forme ou par le repérage afin d'observer ou d'analyser des notions en particulier (ex. : identifier le vers où se trouve tel procédé langagier ou tel thème). L'interprétation est travaillée, mais dans une moindre mesure que la compréhension; par exemple, au $2^{\mathrm{e}}$ cycle, les élèves doivent répondre à une centaine de questions dans chaque manuel, dont $75 \%$ visent la compréhension.

$20 \mathrm{Au} 1^{\mathrm{er}}$ cycle, la section sur la poésie se termine toujours par une production - écrite ou orale - à réaliser, ce qui est moins présent au $2^{\mathrm{e}}$ cycle où l'accent est plutôt mis sur la lecture. Dans tous les cas, les tâches en écriture relèvent de l'imitation (écrire à la manière de) ou impose des contraintes quant au thème, au nombre de figures de style et de vers à insérer, etc., afin de mettre à profit les savoirs étudiés. Ces pratiques d'écriture proviennent surtout d'une approche traditionnelle de l'enseignement de la poésie : les textes sont utilisés comme des modèles à apprécier et à imiter, ou desquels s'inspirer fortement en vue d'écrire une poésie plus personnelle. Quant à l'oral, aucun 
savoir sur la voix ou le corps n'est explicité ou ne fait l'objet d'exercices et de rares tâches portent sur la récitation ou le slam, genres oraux nommés, mais non décrits.

\section{La poésie dans les manuels : des textes à analyser et à imiter}

21 Les manuels visent surtout à former des lecteurs inscrits dans une posture distanciée, analytique, la présence du sujet lecteur apparaissant faiblement sinon dans les quelques questions d'appréciation et de réaction. Quant au sujet scripteur et performeur, il est absent des manuels étant donné la forte prégnance de l'imitation; la créativité des élèves est peu encouragée, et encore moins chez les plus jeunes, ce qui entre en contradiction avec les programmes qui invitent d'abord à les familiariser à la poésie par les jeux de langage en convoquant leur psychoaffectivité. On vise dans ces manuels à ce que les élèves soient capables de reproduire des procédés langagiers, des structures et des jeux de sonorités selon une thématique donnée : le poème est un texte à imiter afin de montrer l'appropriation d'un genre (la fable ou le haïku, surtout) ou du style d'un auteur. Cela dit, quelques propositions plus novatrices apparaissent, comme demander aux élèves d'écrire en équipe, de slamer ou de participer à des manifestations culturelles.

\section{Le discours des passeurs de poésie}

Nous présentons les pratiques déclarées d'enseignement de la poésie en nous attardant aux savoirs et tâches présentés aux élèves, aux finalités visées, aux limites que les enseignants disent rencontrer et à quelques pratiques d'enseignement alternatives qui ouvrent de nouveaux horizons pour la poésie à l'école.

\section{Savoirs et tâches privilégiés dans les enseignements}

Du côté des savoirs enseignés, comme dans les manuels, trois notions reviennent systématiquement, peu importe le degré et la manière de les présenter: les types de rime, les règles de versification et les figures de style. En revanche, les enseignants du $1^{\text {er }}$ cycle insistent davantage sur le travail lexical, les élèves étant encore à parfaire leurs habiletés en lecture, alors qu'au $2^{\mathrm{e}}$ cycle, une place plus importante est accordée aux savoirs historiques ainsi qu'aux notions de thèse et de point de vue, caractéristiques spécifiques de la poésie engagée.

Quant aux tâches, les enseignants du $1^{\text {er }}$ cycle privilégient surtout des dispositifs canoniques : écriture à la manière de, lecture expliquée de poèmes, questions visant à $\mathrm{y}$ repérer des figures de style puis à les relier à leur définition, scander, etc. Au $2^{\mathrm{e}}$ cycle, la poésie permettrait d'aiguiser les compétences analytiques, réflexives et critiques: l'écriture d'imitation et les examens de connaissances y sont également présents, mais les analyses littéraires et appréciations critiques s'ajoutent. La principale distinction entre les cycles concerne l'oral : au $1^{\mathrm{er}}$ cycle, les enseignants restreignent les élèves à une posture d'écoute de poèmes ou chansons alors qu'au $2^{\mathrm{e}}$ cycle, ils leur demandent d'écouter des chansons et slams, mais aussi d'en écrire, voire parfois de performer leur slam, et ce, même si ce n'est pas prescrit par les programmes, et sans forcément enseigner les caractéristiques vocales ou corporelles de la performance (ÉmeryBruneau \& Brunel, 2016). Or, il s'agit là d'une pratique plutôt récente étant donné que les joutes de slam n'existent au Québec que depuis 2006 (Émery-Bruneau \& Yobé, 2015). 


\section{Ruptures, continuités et freins dans les pratiques des passeurs de poésie}

25 stylistiques et formels, à quelques genres (haïku, sonnet, poèmes engagés, chanson et slam), à une pratique scripturale d'imitation et à une pratique lectorale distanciée, analytique et complexe (explication de texte, analyse littéraire, interprétation). Dans tous les cas, nous observons une faible présence accordée à l'émotion poétique ou au poème du lecteur (Favriaud, 2011). Pour les enseignants, la poésie permet d'inculquer des notions évaluables ou de développer des compétences surtout scripturales. Leur vision de la poésie croise les finalités qu'ils poursuivent, car lorsque nous les avons interrogés sur la pertinence d'enseigner la poésie, deux finalités ressortent : 1) la poésie permet de développer la culture, car les élèves n'ont pas ou peu de pratiques poétiques en dehors de l'école, selon eux, hormis l'écoute de chansons - du reste, rarement francophones; 2) la poésie permet de développer les compétences langagières des élèves, dont l'écriture d'imitation et la lecture expliquée.

Or, s'ils n'accordent pas un véritable espace au poème du lecteur comme l'encouragent les didacticiens, c'est peut-être aussi parce qu'ils déclarent se sentir eux-mêmes peu outillés et même freinés pour enseigner la poésie. En les interrogeant, nous avons été en mesure d'identifier au moins cinq explications à ces limites. 1- Seules deux des 20 enseignants disent avoir des pratiques poétiques personnelles régulières, lesquelles nourrissent leurs réflexions sur les différentes manières de l'enseigner; les autres se sentent peu enclins à expérimenter la poésie autrement que de la manière dont ils l'ont eux-mêmes étudiée dans leur parcours scolaire, comme l'une d'entre eux le confie: «j'avoue qu'il y a peut-être ma confortabilité [...] Je ne suis pas une grande consommatrice de [poésie] alors c'est difficile de transmettre ça après ». 2- Les limites matérielles entrainent aussi un important écart vis-à-vis des pratiques sociales poétiques: des enseignants dénoncent qu'il n'y a pas de recueils de poésie à leur disposition (la loi sur l'instruction publique ne permet pas d'en faire acheter par les parents) contrairement aux séries de romans, alors ils travaillent avec les manuels ou photocopient quelques poèmes à lire en fonction des tâches à réaliser. 3- Tous les enseignants s'imposent de terminer leur séquence sur la poésie par une ou même deux évaluations (production écrite à la manière de..., examen de connaissances), ce qui nous rappelle la critique de G. Fourez (2003) qui dénonce que les systèmes scolaires sont de plus en plus conditionnés par l'évaluation, reléguant au second rang les conditions d'apprentissage, le développement du plein potentiel des élèves et la formation des enseignants. Alors comment pourrait-on mesurer les émotions poétiques ou évaluer un poème du lecteur? Les pratiques traditionnelles comme le repérage des figures de style et l'écriture d'imitation ou à contrainte servent l'évaluation, car elles offrent des critères objectifs et infaillibles pour juger les capacités des élèves (ex.: Voit-on les mêmes caractéristiques de la fable J. de la Fontaine dans la production de l'élève? Retrouve-t-on dans le texte de l'élève les trois figures de style exigées, un champ lexical sur le thème choisi, la forme du sonnet, les alexandrins et les rimes croisées ?). Or, ces pièges de l'évaluation conduisent à se demander si ce qui est évalué par les enseignants est davantage tourné vers les capacités des élèves à imiter, reproduire ou repérer plutôt que celles d'interpréter, de créer ou de performer poétiquement... L'un des enseignants est très réaliste à cet effet : «La formule qu'on enseigne [et] qu'on évalue [...] est "je te montre une façon d'écrire" [...] c'est de la logique [mais] en même temps il 
n'y a pas de place à la créativité là-dedans pis on ne la permettra pas tant que ça ni au cégep $^{4}$ ni à l'université cette créativité, on veut un rapport [formel]. Alors, je pense que des fois [l'évaluation] peut être un frein ». 4- La formation initiale insuffisante a aussi été dénoncée par quelques enseignants: "Je ne me souviens pas avoir fait une formation précise sur l'enseignement de la poésie ", ce qui va dans le même sens que ce que nous avons décrit dans un autre article (Émery-Bruneau \& Leclerc, 2018). 5- Le manque de formation continue souligné entre autres par une enseignante qui affirme voir très peu d'ateliers sur la poésie dans les congrès professionnels : « On va parler de grammaire, grammaire, grammaire. Beaucoup de grammaire. Mais poésie, très peu. » Pourtant cette enseignante de $1^{\text {re }}$ secondaire a soulevé ce paradoxe : «Quand on voit que c'est un des quatre genres qu'on doit enseigner... C'est le quart! Est-ce qu'en toute logique, il faudrait passer le quart de notre temps [à enseigner la poésie] ? »

\section{Pratiques alternatives et nouveaux horizons pour enseigner la poésie}

Malgré ces limites que rencontrent les enseignants, et bien que de nombreuses pratiques semblent cristallisées dans des tâches traditionnelles, quelques-uns choisissent de travailler la poésie autrement. Rappelons que les deux genres privilégiés sont actuels : la chanson contemporaine et le slam. Le slam est d'ailleurs un genre qui a participé à une révolution tranquille de la poésie (Émery-Bruneau \& Yobé, 2015) : six des 20 enseignants de notre étude ont mentionné avoir déjà assisté à une joute de slam et tous les enseignants de $4^{\mathrm{e}}$ secondaire interrogés présentent le slam en classe, bien que dans tous les autres degrés, des enseignants disent aussi l'intégrer à leur planification (Émery-Bruneau, 2018). Aussi, tous les enseignants du $2^{\mathrm{e}}$ cycle travaillent des chansons en classe, genre plus près de la culture des élèves selon eux. La prise en compte d'une poésie contemporaine, oralisée et performée, présente manifestement depuis une décennie une innovation dans l'enseignement de la poésie au secondaire québécois.

Il en est de même de la lecture subjective, plus encouragée par les enseignants qui invitent leurs élèves à découvrir différents poèmes ou à cheminer dans leurs appréciations, comme celle-ci : «Moi, c'est vraiment ça : tu découvres! Sans jugement. Ouverture d'esprit. T'aimes ça, t'aimes pas ça : ce n'est pas grave. Au moins ça te fait ça de plus dans ton bagage. " Même si la lecture expliquée et le repérage demeurent des pratiques répandues, et même si l'admiration des canons est encore très présente, le sujet lecteur semble de plus en plus autorisé à exprimer ses émotions poétiques.

\section{Finalités de l'enseignement de la poésie et propositions didactiques}

Alors comment transformerait-on l'enseignement traditionnel de la poésie de façon à se rapprocher à la fois des pratiques d'enseignement alternatives et des pratiques sociales de référence pour permettre aux élèves de vivre des expériences poétiques variées et subjectives, puis de les inviter à partager ces évènements intimes et à en être ultimement transformés comme sujets, élèves et citoyens? Nous proposons cinq pistes didactiques pour y arriver, lesquelles sont complémentaires et organisées selon une progression spiralaire pour bonifier les expériences poétiques vécues par les élèves. Cela dit, aucune ne doit être exclue ou exclusive, car l'ordre dans lesquelles les utiliser 
variera selon le contexte d'enseignement, le moment de l'année scolaire, les textes choisis, les intentions didactiques de l'enseignant, les capacités des élèves, etc.

$1^{\circ}$ Habiter le poème - par la lecture subjective, la réécriture ou la performance - pour en faire son poème de lecteur.

Il s'agit d'inscrire les élèves dans une posture de lecture créative afin de les amener à s'investir subjectivement. Habiter le poème signifie leur demander de s'interroger sur leur façon de dialoguer avec le texte afin de comprendre ce qu'ils en aiment, ce qui les rebute, ce qu'ils trouvent confortable, intéressant, agréable dans le poème, ce qu'ils ne comprennent pas, ce qu'ils en retiennent après coup, etc., puis d'exprimer leurs réactions. Les dispositifs didactiques pour y arriver sont nombreux, dont les groupes de discussion ou les cercles de lecture pour partager les réceptions singulières; le recueil à quatre mains (Brillant-Rannou, 2016) pour annoter le texte de ses réactions ou interprétations ; les performances poétiques pour inscrire leur voix et leur corps dans le poème (Émery-Bruneau \& Brunel, 2016); ou la réécriture d'un poème en répondant à une consigne du type "Réécrivez ce poème en le transformant suffisamment pour exprimer à votre manière ce que vous auriez voulu lire. Faites de ce poème le vôtre " (Émery-Bruneau \& Leclerc, 2018) pour les amener à créer leur poème de lecteur.

$2^{\circ}$ Cheminer à travers les expériences poétiques pour progresser en tant que sujet lecteur/ scripteur/performeur.

Il s'agit d'inscrire les élèves dans une posture réflexive pour les amener à prendre conscience de leurs émotions poétiques, de leur façon de (re)vivre des expériences poétiques, de comparer ces expériences et de les commenter pour se constituer comme sujet, conscient de sa progression en regard de ce qu'il comprend, interprète, crée, écrit ou de la manière dont il incarne une performance poétique d'une fois à l'autre. Afin de les amener à comparer ce qu'ils vivent d'une expérience poétique à l'autre, à exprimer ce qu'ils observent, apprennent, interprètent, ressentent différemment, les dispositifs didactiques suivants offrent un réel potentiel: de courts débats interprétatifs pour affiner leurs interprétations, leurs expériences et leurs réflexions; la formulation de commentaires écrits - dans des carnets de lecture par exemple - ou oraux - dans des groupes de réflexion - sur différents poèmes lus ou performances poétiques écoutées ou visionnées; la création d'une partition pour réfléchir à leur expérience d'écoute analytique ou de performance poétique à incarner par un travail conscient de la voix et du corps (Émery-Bruneau \& Brunel, 2016); la production d'écrits intermédiaires (Bucheton \& Chabanne, 2002) lors d'exercices d'écriture créative ou d'oraux intermédiaires préparant à la performance, par exemple, lors d'ateliers d'écriture ou de cercles de performances (sous-groupe) dans lesquels les élèves font différents exercices de style ou s'exercent à performer et donnent de la rétroaction à leurs pairs afin qu'ils bonifient leur production.

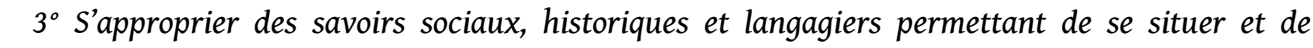
s'orienter par rapport aux pratiques poétiques.

L'école est un cercle de culture seconde (Simard, 2005) permettant de faire des apprentissages conscients à l'aide de savoirs sélectionnés en fonction des disciplines, lesquels facilitent notre compréhension du monde, de soi et des autres. Il nous apparait par conséquent important de rappeler les trois types de savoirs proposés par K. Canvat et G. Legros (1997), tout en les actualisant, puis en les articulant à nos quatre autres propositions. 
36 Afin de permettre aux élèves d'avoir des référents culturels et épistémiques pour se situer par rapport aux genres poétiques, poètes, pratiques poétiques, etc. auxquels ils sont exposés, trois types de savoirs sont à enseigner. D'une part, les savoirs sociaux, soit ceux qui concernent le champ littéraire (les maisons d'édition de recueils de poésie, les festivals de poésie, les poètes canonisés et ceux de la relève, les préfaces/ postfaces de recueils, etc.), l'image des poètes en fonction de leurs contextes de production (processus de création, intention de l'auteur, discours méta sur la poésie, etc.), les pratiques sociales de référence (soirée de poésie, joutes de slam, performances poétiques, attentat poétique, etc.), les modes et opérations de lecture (participation, distanciation, interprétation, appréciation...), etc. D’autre part, les savoirs historiques, qui permettent de mieux comprendre les contextes de production des poèmes, les contextes de création ou de performance des poètes, soit les courants littéraires, l'histoire d'un mouvement artistique, l'histoire d'une communauté de poètes, etc. Enfin, les savoirs langagiers et méta-langagiers, car la poésie est aussi un langage enseigné dans une classe de français: il s'agit donc des savoirs syntaxiques, sémantiques, métriques, génériques, rhétoriques, typographiques, etc.

$4^{\circ}$ Apprécier différentes manifestations culturelles (ce qui inclut les perspectives sociales et historiques de la poésie).

Il s'agit aussi d'amener les élèves à poser un jugement ou à évaluer une performance poétique vue ou entendue, un recueil de poèmes lu, un exercice d'écriture créative vécu, etc., pour qu'ils passent du jugement de gout au jugement esthétique. Pour ce faire, ils peuvent apprendre à convoquer quatre dimensions axiologiques fondamentales (Brunel et al., 2018, p. 281) pour exprimer leur jugement sur un texte, une performance ou une expérience poétique : la cognition, à savoir leurs normes de lisibilité pour poser leur jugement ; l'éthique, soit leurs normes morales ou idéologiques pour défendre leur critique; les références socioculturelles, soit les repères qui leur servent de guide pour apprécier le poème lu ou entendu ; et l'esthétique, soit les normes stylistiques pour poser un jugement réfléchi sur la poésie. Outre les traditionnelles activités d'écriture de type analyse littéraire ou compte rendu critique, d'autres dispositifs didactiques permettraient d'atteindre ces objectifs, par exemple la production vidéo d'une bande-annonce pour promouvoir une soirée de poésie ou une joute de slam; la diffusion d'un message publicitaire (audio ou vidéo) d'un recueil de poésie pour inciter les gens à le lire; l'animation d'une table ronde sur des poètes contemporains de la relève, etc.

$5^{\circ}$ Prendre conscience des transformations de son rapport aux savoirs, à la langue, à l'expérience poétique...

Nous l'avons vu, avec la poésie, la progression est moins du côté de l'objet et davantage du côté des compétences langagières et culturelles à (faire) développer, ainsi que des expériences poétiques à (faire) vivre comme sujets lecteurs, scripteurs ou performeurs. Par conséquent, il s'agit d'amener les élèves à objectiver les transformations de leur rapport à la poésie, par exemple, en expliquant leur démarche de création ou en justifiant ce qu'ils visent à créer comme effet dans leur performance poétique ou dans un exercice d'écriture créative ; en commentant l'annotation qu'ils font sur des poèmes auxquels ils réagissent ou qu'ils interprètent ; en faisant un exposé oral pour présenter une création qu'ils ont faite ou pour décrire les effets d'expériences poétiques sur leur façon de comprendre un poète, un thème, un rythme, etc. 


\section{Conclusion}

Il nous semble essentiel de transformer les pratiques traditionnelles d'enseignement de la poésie, cristallisées depuis des décennies dans l'admiration, l'imitation et la reproduction, et de réfléchir avec les enseignants, futurs enseignants et formateurs d'enseignants à leur rapport à la poésie, afin de créer un pont entre les pratiques ordinaires, les pratiques alternatives et les ingénieries des didacticiens de la littérature. En plus des propositions pour la formation initiale et continue des enseignants que nous avions émises ailleurs (Émery-Bruneau \& Leclerc, 2018), l'ingénierie didactique collaborative pourrait ainsi être un type de recherche à retenir pour les prochains chantiers en didactique de la littérature qui participeront au renouvèlement de l'enseignement de la poésie, ainsi qu'à une profonde réflexion sur les finalités de son enseignement. Partant, reprendre nos cinq propositions didactiques et les expérimenter avec des enseignants des différents cycles/degrés nous apparait être un premier pas vers des finalités transformées de l'enseignement de la poésie et pensées en fonction de la progression, point nodal de la didactique.

\section{BIBLIOGRAPHIE}

BOUTEVIN, C. (2014). Le livre de poème(s) illustré. Étude d'une production littéraire en France de 1995 à nos jours et de sa réception par les professeurs des écoles. Thèse en littérature française, Université Grenoble 3.

BRILLANT-RANNOU, N. (2010). Le lecteur et son poème. Lire en Poésie : expérience littéraire et enjeux pour l'enseignement du français en lycée. Thèse en didactique de la littérature, Université Rennes 2. BRILLANT-RANNOU, N. (2016). « Le recueil à quatre mains et la lecture dialoguée dans les marges : conception et expérimentation de deux dispositifs de lecture subjective de poésie ». In : BrillantRannou, N., Boutevin, C. \& Brunel, M. (dirs), Être et devenir lecteur(s) de poèmes. De la poésie patrimoniale au numérique. Namur : Presses universitaires de Namur, p. 87-109.

BRUNEL, M. \& ÉMERY-BRUNEAU, J. (2016). « Réciter, dire, écouter, agir : tensions et écarts dans les enjeux de la performance poétique dans l'enseignement secondaire en France et au Québec ». In : Brillant-Rannou, N., Boutevin, C. \& Brunel, M. (dirs), Être et devenir lecteur(s) de poème. De la poésie patrimoniale au numérique. Namur : Presses universitaires de Namur, p. 197-216.

BRUNEL, M. et al. (2018). « Le discours des élèves sur les valeurs du texte littéraire et leur exploitation didactique par les enseignants : quelles variations selon les classes d'âge et selon les pays ? ». In : Rouvière, N. (dir.), Enseigner la littérature en questionnant les valeurs. Bruxelles :

P. Lang, p. 279-302.

BUCHETON, D. \& CHABANNE, J.-C. (2002). Parler et écrire pour penser, apprendre et se construire. Paris : Presses universitaires de France.

CANVAT, K. \& Legros, G. (1997). « Enseigner la poésie moderne ? ». Pratiques 96, p. 5-29. En ligne : https://www.persee.fr/doc/prati_0338-2389_1997_num_93_1_1792. 
COLlot, M. (2005) [1989]. La poésie moderne et la structure d'horizon. Paris : Presses universitaires de France.

DELAS, D. (1973). Linguistique et poétique. Paris : Larousse.

DUfAYS, J.-L., GEMENNE, L. \& LEDUR, D. (2015) [1996]. Pour une lecture littéraire. Louvain-la-Neuve : De Boeck.

DUfAYS, J.-L., LISSE, M. \& MEURÉE, C. (2009). Théorie de la littérature. Louvain-la-Neuve : AcademiaBruylant.

DUVAL, I., \& TURCOTTE, M. (2007). « Dynamiser l'enseignement de la poésie contemporaine ». Québec français 147, p. 62-64. En ligne : https://www.erudit.org/fr/revues/qf/2007-n147qf1176972/45591ac.pdf.

ÉMERY-BRUNEAU, J. (2018). « Le rapport à la poésie d'enseignants du secondaire québécois : quelle progression entre les cycles/degrés ?». Tréma 49, p. 29-42. En ligne : https://

journals.openedition.org/trema/4526.

ÉMERY-BRUNEAU, J. \& BRUNEL, M. (2016). « Poésie oralisée et performée : quel objet, quels savoirs, quels enseignements? ». Repères 54, p. 189-206. En ligne : https://journals.openedition.org/ reperes/1117.

ÉMERY-BRUNEAU, J. \& LECLERC, C. S. (2018). « Expériences poétiques d'étudiants en formation initiale en enseignement du français au secondaire québécois ». Language and Literacy 20(2), p. 20-39.

ÉMERY-BRUNEAU, J. \& YOBÉ, V. (2015). « La joute de slam : description de la pratique sociale et analyse de sa transposition didactique ». In : Lafontaine, L. \& Pharand, J. (dirs), Littératie. Vers une maitrise des compétences dans divers environnements. Québec : Presses de l'Université du Québec, p. $185-206$.

FAVRIAUD, M. (2011) « Le poème du lecteur : mémorisation, imagination, compréhension - et image de soi ». In : Mazauric, C., Fourtanier, M.-J. \& Langlade, G. (éds), Le texte du lecteur. Bruxelles : P. Lang, p. 175-192.

FAVRIAUD, M., VinsonNeAu, M. \& Poletto, M. (2017). Les Chemins de poésie d'Alep. Poétique et didactique du dire-lire-écrire à l'école primaire. Limoges : Lambert-Lucas.

FOUREZ, G. (2003). « Évaluation, relations pédagogiques, rôle de l'erreur ». La Revue nouvelle 5-6, p. 75-89. En ligne : http://www.revuenouvelle.be/IMG/pdf/075-089_ARTICLE_Fourez.pdf.

MARTIN, S. (2010). « Présentation. Les poèmes au cœur de l'enseignement du français ». Le français aujourd'hui 2 (169), p. 3-14.

MINISTÈRE DE L'ÉDUCATION DU QUÉBEC (MÉQ). (2005). Programme de formation de l'école québécoise. Enseignement secondaire, premier cycle. Québec : Gouvernement du Québec.

MINISTÈRE DE L'ÉDUCATION, DU LOISIR ET DU SPORT DU QUÉBEC (MÉLS). (2009). Programme de français, langue d'enseignement, du deuxième cycle du secondaire. Québec : Gouvernement du Québec.

MINISTÈRE DE L'ÉDUCATION, DU LOISIR ET DU SPORT DU QUÉBEC (MÉLS). (2011). Progression des apprentissages au secondaire. Français, langue d'enseignement. Québec : Gouvernement du Québec.

NONNON, É. (2010). « La notion de progression au cœur des tensions de l'activité d'enseignement ». Repères 41, p. 5-34. En ligne : https://journals.openedition.org/reperes/276.

SIMARD, D. (2005). «Comment penser aujourd'hui la nature et le rôle de l'école à l'égard de la formation culturelle des élèves ?». In : Simard, D. \& Mellouki, M. (éds), L'enseignement, profession intellectuelle. Québec : Presses universitaires de Laval, p. 49-74. 


\section{NOTES}

1. Nous remercions le Conseil canadien de recherche en sciences humaines (CRSH) pour son financement.

2. Il s'agit de tous les manuels approuvés officiellement par le ministère de l'Éducation du Québec, lesquels ne sont toutefois pas conçus par des didacticiens : http://www1.education.gouv.qc.ca/ bamd/doc/Liste_secondaire_fr_new.pdf. Les enseignants peuvent choisir d'utiliser un de ces manuels pour leur degré d'enseignement ; leur choix est personnel ou collectif (entente avec une équipe d'enseignants d'une même école).

3. Au $1^{\text {er }}$ cycle, les élèves sont âgés en moyenne de 12 à 14 ans ; au $2^{\mathrm{e}}$ cycle, ils ont de 14 à 17 ans.

4. Le cégep renvoie aux années d'études postsecondaires : la filière préuniversitaire est réalisée en deux ans et la filière technique en trois ans. Peu importe la filière et le programme, les quatre cours obligatoires de littérature (française et québécoise) doivent être réussis pour être diplômé.

\section{RÉSUMÉS}

Nous présentons l'analyse de trois discours qui marquent la manière d'enseigner et d'apprendre la poésie au sein de la forme scolaire québécoise : les programmes du secondaire, les manuels (corpus, savoirs et tâches), ainsi que les pratiques déclarées des enseignants (savoirs et tâches présentés aux élèves, finalités visées, freins ou limites vécues, pratiques alternatives). Ce croisement des discours permet de mieux saisir les dimensions de la poésie enseignée et les dispositifs didactiques les plus répandus en fonction des cycles/degrés pour observer la progression de cet objet d'enseignement. Les ruptures, continuités et points aveugles observés, ainsi que les quelques pratiques alternatives relevées, lesquelles tentent de s'affranchir d'un enseignement traditionnel dominant, nous ont amenée à proposer des pistes didactiques qui participent au renouvèlement de l'enseignement de la poésie et qui conçoivent les élèves en tant que sujets lecteurs, scripteurs et performeurs.

We present an analysis of three discourses that highlight the way poetry is taught and learned in Quebec: high school curricula, textbooks (corpus, knowledge and tasks), as well as teachers' declared practices (knowledge and tasks presented to students, intended purposes, obstacles or limitations, alternative practices). This discourse analysis allows a better grasp of the configuration of the poetry taught and the most widespread didactic devices according to cycles/ degrees to observe the progression of this object taught. The ruptures, continuities and blind spots observed, as well as the few alternative practices identified, which attempt to break away from the dominant traditional teaching of poetry, have led us to propose didactic approaches that participate in the renewal of the teaching of poetry and that holding students as subjective readers, authors and performers. 
INDEX

Mots-clés : didactique de la littérature, poésie, progression, finalités de l'enseignement, pratiques d'enseignement, propositions didactiques

Keywords : didactic of literature, poetry, progression, teaching practices, didactic propositions

\section{AUTEUR}

JUDITH ÉMERY-BRUNEAU

Université du Québec en Outaouais (Gatineau), J8X 3X7, Canada 